PROCEEDINGS OF THE

AMERICAN MATHEMATICAL SOCIETY

Volume 130, Number 4, Pages 1235-1241

S 0002-9939(01)06157-3

Article electronically published on September 14, 2001

\title{
ON THE RELATION BETWEEN THE A-POLYNOMIAL AND THE JONES POLYNOMIAL
}

\author{
RĂZVAN GELCA
}

(Communicated by Ronald A. Fintushel)

\begin{abstract}
This paper shows that the noncommutative generalization of the A-polynomial of a knot, defined using Kauffman bracket skein modules, together with finitely many colored Jones polynomials, determines the remaining colored Jones polynomials of the knot. It also shows that under certain conditions, satisfied for example by the unknot and the trefoil knot, the noncommutative generalization of the A-polynomial determines all colored Jones polynomials of the knot.
\end{abstract}

\section{INTRODUCTION}

In 1984, V. Jones introduced a polynomial invariant of knots 8] through skein relations. Another version of this invariant, the Kauffman bracket 9], was introduced shortly after. Colored versions of these invariants were defined, via quantum groups [12, and via Jones-Wenzl idempotents [10], 1].

In 1993, Cooper, Culler, Gillet, Long, and Shalen defined a two variable polynomial invariant of knots, the A-polynomial, using the character variety of $S L(2, \mathbb{C})$ representations of the fundamental group of the knot complement. This invariant was generalized in [5] to a finitely generated ideal of polynomials in the quantum plane, the A-ideal. This is done in the context of skein modules, and is based on the fact that the Kauffman bracket skein modules represent deformations of function rings on character varieties [2, 11, and on the relationship between the skein algebra of the cylinder over a torus and the noncommutative torus [4]. The noncommutative A-ideal depends on a deformation parameter $t$; specializing $t=-1$ one recovers the classical A-polynomial as the generator of the radical of the one dimensional part of this ideal.

As shown in [5], each element in the noncommutative A-ideal defines a matrix that annihilates the vector whose entries are the colored Jones polynomials of the knot (or, more precisely, the colored Kauffman brackets of the knot; they differ from the colored Jones polynomials by the change of variable $t \mapsto i t)$. The orthogonality between the rows of the matrix and the vector whose entries are the colored Jones polynomials of the knot has been called the "orthogonality relation (between the Jones polynomial and the A-polynomial)".

Received by the editors May 9, 2000 and, in revised form, October 23, 2000.

1991 Mathematics Subject Classification. Primary 57M25, 58B30, 46L87.

Key words and phrases. Kauffman bracket, Jones polynomial, A-polynomial, noncommutative geometry. 
The present paper shows that the noncommutative A-ideal together with a finite number (depending on the A-ideal of the knot) of colored Kauffman brackets of the knot determine all other colored Kauffman brackets of the knot. We also prove that under certain technical conditions on the A-ideal, the noncommutative Aideal determines all colored Kauffman brackets of the knot. For example, any knot having the same A-ideal as the unknot, respectively the trefoil knot, has the same colored Kauffman brackets as the unknot, respectively the trefoil knot.

A long standing question regarding the Jones polynomial is how it relates to the $S U(2)$-representations of the fundamental group of the knot complement. The A-polynomial was defined using the variety of $S L(2)$-representations of the fundamental group, and the noncommutative A-ideal was introduced as a noncommutative generalization of this polynomial. By showing that the noncommutative A-ideal virtually determines the Jones polynomial and its colored versions, this paper intends to shed some light on the relationship between this polynomial and the representations of the fundamental group.

\section{The ACTION OF $K_{t}\left(\mathbb{T}^{2} \times I\right)$ ON $K_{t}\left(\mathbb{D}^{2} \times I\right)$}

The Kauffman bracket skein module of the three manifold $M$ is defined in the following way. Let $\mathbb{C}\left[t, t^{-1}\right] \mathcal{L}$ be the $\mathbb{C}\left[t, t^{-1}\right]$-module freely spanned by the isotopy classes of framed links in $M$ including the empty link, and let $\mathcal{S}$ be the submodule spanned by the relations $\left.\backslash-t \cup-t^{-1}\right)\left(\right.$ and $\bigcirc+t^{2}+t^{-2}$. The Kauffman bracket skein module of $M$ is $K_{t}(M)=\mathbb{C}[t] / \mathcal{S}$.

In the case where $M$ is the cylinder over a surface, $K_{t}(M)$ has a natural algebra structure, with product defined by placing one link on top of another. If $M$ is a manifold with boundary, the operation of gluing a cylinder to the boundary induces a $K_{t}(\partial M \times I)$-module structure on $K_{t}(M)$. As an example it is known that the Kauffman bracket skein algebra of the cylinder over an annulus (i.e., that of the solid torus) is $\mathbb{C}\left[t, t^{-1}, \alpha\right]$, where $\alpha$ is the curve that runs once around the annulus and has framing parallel to the annulus.

Another, more complicated example is that of the Kauffman bracket skein algebra of $K_{t}\left(\mathbb{T}^{2} \times I\right)$. Its multiplication rule and action on the skein module of the solid torus are described by means of two families of Chebyshev polynomials, $\left\{T_{n}\right\}_{n \in \mathbb{Z}}$ defined by $T_{0}=2, T_{1}=x, T_{n+1}=x T_{n}-T_{n-1}$ for $n \in \mathbb{Z}$ and $\left\{S_{n}\right\}_{n \in \mathbb{Z}}$ defined by $S_{0}=1, S_{1}=x, S_{n+1}=x S_{n}-S_{n-1}$ for $n \in \mathbb{Z}$. The two polynomials are defined by the same recurrence relation, but the initial conditions are different. Note that $T_{-n}=T_{n}$ and $S_{-n}=-S_{n-2}$, for all $n \in \mathbb{Z}$. Let $p$ and $q$ be two integers with $p=n p^{\prime}, q=n q^{\prime}, p^{\prime}, q^{\prime}$ coprime. We define $(p, q)_{T}=T_{n}\left(\left(p^{\prime}, q^{\prime}\right)\right)$, where $\left(p^{\prime}, q^{\prime}\right)$ is the corresponding curve on the torus, with framing parallel to the torus, and its powers are defined by taking parallel copies. The elements $(p, q)_{T}, p \geq 0, q \in \mathbb{Z}$ span $K_{t}(M)$ as a $\mathbb{C}\left[t, t^{-1}\right]$-module. In [4] the following product-to-sum formula was proved:

$$
(p, q)_{T} *(r, s)_{T}=t^{\mid p q} \mid(p+r, q+s)_{T}+t^{-\left.\right|_{r s} ^{p q} \mid}(p-r, q-s)_{T}
$$

As a consequence of this formula, the Kauffman bracket skein algebra of the cylinder over a torus is isomorphic to the subalgebra of the noncommutative torus generated by noncommutative cosines. Let us recall that the algebra of trigonometric polynomials in the noncommutative torus is $\mathbb{C}_{t}\left[l, l^{-1}, m, m^{-1}\right]$, with multiplication $*$, satisfying $l * m=t^{2} m * l$. The elements $e_{p, q}=t^{-p q} l^{p} m^{q}$ are the 
noncommutative exponentials; they satisfy

$$
e_{p, q} * e_{r, s}=t^{\mid p_{r s} q} e_{p+r, q+s} .
$$

The noncommutative cosines are $\frac{1}{2}\left(e_{p, q}+e_{-p,-q}\right)$. The map $(p, q)_{T} \rightarrow e_{p, q}+e_{-p,-q}$ gives the isomorphism between $K_{t}\left(\mathbb{T}^{2} \times I\right)$ and the algebra of noncommutative cosines.

Let $K$ be a knot in $S^{3}$, and let $M$ be the complement of a regular neighborhood of $K$. Consider the left action of $K_{t}\left(\mathbb{T}^{2} \times I\right)$ on $K_{t}(M)$ defined by gluing the cylinder over the torus to the boundary of $M$ such that the curve $(1,0)$ is identified with the longitude of the knot and the curve $(0,1)$ is identified with the meridian. The peripheral ideal of $K$, denoted by $I_{t}(K)$, is the left ideal of $K_{t}\left(\mathbb{T}^{2} \times I\right)$ which annihilates the empty skein in the knot complement. The noncommutative Aideal of $K$, denoted by $\mathcal{A}_{t}(K)$, is the left ideal obtained by extending $I_{t}(K)$ to $\mathbb{C}_{t}\left[l, l^{-1}, m, m^{-1}\right]$ and then contracting it to $\mathbb{C}_{t}[l, m]$. As explained in [5], the Aideal is a noncommutative generalization of the A-polynomial. The A-polynomial is obtained by setting $t=-1$, replacing $l$ and $m$ by $-l$ and $-m$, and taking the generator of the radical of the one-dimensional part of the A-ideal (divided by $(l-1))$. The operation of extending and restricting ideals from $K_{t}\left(\mathbb{T}^{2} \times I\right)$ to obtain ideals in $\mathbb{C}_{t}[l, m]$ is a one-to-one correspondence $[5$. Why then work with the Aideal and not with the peripheral ideal? The reason is that ideals of polynomials, even in the noncommutative setting, are easier to understand and to work with, and there exists a large amount of computer software for them.

There is a left and a right action of $K_{t}\left(\mathbb{T}^{2} \times I\right)$ on $K_{t}\left(\mathbb{D}^{2} \times S^{1}\right)$, one for the positive and the other for the negative orientation of the boundary torus. To understand them, let us denote by $x_{p, q}$ the image in $K_{t}\left(\mathbb{D}^{2} \times S^{1}\right)$ of $(p, q)_{T}$ on the boundary torus (with the positive orientation). It is not hard to see that $x_{0, q}=\left(-t^{2}\right)^{q}+\left(-t^{-2}\right)^{q}$ and the product-to-sum formula yields

$$
x_{p+1, q}=t^{-q}(1,0) \cdot x_{p, q}-t^{-2 q} x_{p-1, q} .
$$

The second order recursive relation for $t^{p q} x_{p, q}$ has fixed coefficients, and hence a formula for the general term can be found. It is

$$
x_{p, q}=t^{-p q}\left(\left(-t^{-2}\right)^{q} S_{p}(\alpha)-\left(-t^{2}\right)^{q} S_{p-2}(\alpha)\right) .
$$

Lifting the skeins $T_{n}(\alpha)$ to the elements $(n, 0)_{T}$ in the boundary torus and using the product-to-sum formula we get the following

Lemma 1. The left action is described by

$$
\begin{aligned}
(p, q)_{T} \cdot T_{n}(\alpha)= & t^{-(2 n+p) q}\left[\left(-t^{-2}\right)^{q} S_{n+p}(\alpha)-\left(-t^{2}\right)^{q} S_{n+p-2}(\alpha)\right] \\
& +t^{(2 n-p) q}\left[\left(-t^{-2}\right)^{q} S_{p-n}(\alpha)-\left(-t^{2}\right)^{q} S_{p-n-2}(\alpha)\right]
\end{aligned}
$$

while the right action is given by

$$
\begin{aligned}
T_{n}(\alpha) \cdot(p, q)_{T}= & (p,-q)_{T} \cdot T_{n}(\alpha) \\
= & t^{(2 n+p) q}\left[\left(-t^{2}\right)^{q} S_{p+n}(\alpha)-\left(-t^{-2}\right)^{q} S_{p+n-2}(\alpha)\right] \\
& +t^{-(2 n-p) q}\left[\left(-t^{2}\right)^{q} S_{p-n}(\alpha)-\left(-t^{-2}\right)^{q} S_{p-n-2}(\alpha)\right] .
\end{aligned}
$$




\section{The Results}

Gluing a solid torus to the complement $M$ of a regular neighborhood of a knot $K$, in such a way that the longitude is glued to the longitude and the meridian to the meridian, induces a pairing

$$
K_{t}\left(\mathbb{D} \times S^{1}\right) \times K_{t}(M) \rightarrow \mathbb{C}\left[t, t^{-1}\right] .
$$

The basis $\left\{S_{n}(\alpha)\right\}_{n}$ induces a family of functionals $\left\langle S_{n}(\alpha), \cdot\right\rangle, n=0,1,2, \ldots$ If we denote by $\emptyset$ the empty link, then

$$
\left\langle S_{n}(\alpha), \emptyset\right\rangle=\kappa_{n}(K),
$$

where $\kappa_{n}(K)$ is the $n$th colored Kauffman bracket of $K$ with zero framing [10], [13] (the $n$th colored Kauffman bracket is a "twisted" version of the $n$th colored Jones polynomial as defined in [12], obtained from the colored Jones polynomials by the change of variable $t \mapsto i t)$. Indeed, the recursive relation for $S_{n}$ shows that the link in $S^{3}$ obtained from the pairing is $K$ colored by the Jones-Wenzl idempotent.

The pairing is compatible with the actions of $K_{t}\left(\mathbb{T}^{2} \times I\right)$ on both modules, i.e. $\left\langle u \cdot(p, q)_{T}, v\right\rangle=\left\langle u,(p, q)_{T} \cdot v\right\rangle$ for any skeins $u$ and $v$. In particular, if $a$ is in the peripheral ideal $I_{t}(K)$ of $K$, then $\langle u \cdot a, \emptyset\rangle=0$. So, if $u=T_{n}(\alpha)$ and $a=\sum_{i} c_{i}\left(p_{i}, q_{i}\right)_{T}$, then by Lemma 1 ,

$$
\begin{aligned}
& \left\langle a \cdot T_{n}(\alpha), \emptyset\right\rangle=\sum_{i} c_{i}\left(t ^ { p _ { i } q _ { i } } \left(t ^ { ( 2 n + p _ { i } ) q _ { i } } \left[\left(-t^{2}\right)^{q_{i}}\left\langle S_{p_{i}+n}(\alpha), \emptyset\right\rangle\right.\right.\right. \\
& \left.-\left(-t^{-2}\right)^{q_{i}}\left\langle S_{p_{i}+n-2}(\alpha), \emptyset\right\rangle\right] \\
& \left.\left.+t^{-\left(2 n-p_{i}\right) q_{i}}\left[\left(-t^{2}\right)^{q_{i}}\left\langle S_{p_{i}-n}(\alpha), \emptyset\right\rangle-\left(-t^{-2}\right)^{q_{i}}\left\langle S_{p_{i}-n-2}(\alpha), \emptyset\right\rangle\right]\right)\right) \\
= & \sum_{i} c_{i}\left(t^{\left(2 n+p_{i}\right) q_{i}}\left[\left(-t^{2}\right)^{q_{i}} \kappa_{p_{i}+n}(K)-\left(-t^{-2}\right)^{q_{i}} \kappa_{p_{i}+n-2}(K)\right]\right. \\
& \left.+t^{-\left(2 n-p_{i}\right) q_{i}}\left[\left(-t^{2}\right)^{q_{i}} \kappa_{p_{i}-n}(K)-\left(-t^{-2}\right)^{q_{i}} \kappa_{p_{i}-n-2}(K)\right]\right)=0 .
\end{aligned}
$$

This relation was called the orthogonality relation in [5] since it expresses the orthogonality between the vector with entries equal to the colored Kauffman brackets of the knot and the rows of the matrix of the linear transformation induced by $a$ from the module $K_{t}(\mathbb{D} \times I)$ with basis $\left\{T_{n}(\alpha)\right\}_{n}$ to the same module with basis $\left\{S_{n}(\alpha)\right\}_{n}$. Since $a$ arises from an element in the noncommutative A-ideal (through an extension and a contraction), orthogonality expresses a relationship between the the elements of the A-ideal and the vector whose entries are the colored Kauffman brackets.

Theorem 1. Let $K$ be a knot and $\psi$ a nonzero element in its peripheral ideal. Then there exists a number $\nu$ depending only on $\psi$ such that $\psi$ together with the first $\nu$ colored Kauffman brackets of the knot determine all the other colored Kauffman brackets of the knot.

Proof. We will show that $\psi$ produces a recursive relation for the colored Kauffman brackets of the knot, whose dominant coefficient is nonzero for large indices. Let $\psi=\sum_{j} c_{j}\left(p_{j}, q_{j}\right)_{T}$ be the element in $I_{t}(K)$, let $p$ be the maximum of $p_{j}$ and assume $p_{j}=p$ if $j=1,2, \cdots, m, p_{j} \neq p$ if $j>m$. Then, the coefficient of $\kappa_{n+p}$ in the orthogonality relation written for $\psi$ is

$$
\sum_{j=1}^{m} c_{j}(-1)^{q_{j}} t^{(2 n+2+p) q_{j}} .
$$


Since the $q_{j}$ 's appearing in this expression are distinct (the $p_{i}$ 's being the same), this expression is identically equal to zero only for finitely many $n$. Consequently the orthogonality relation provides a recursive relation that determines uniquely $\kappa_{n}$ for large $n$.

Corollary 1. For every knot $K$ there is a number $\nu(K)$ such that if $K^{\prime}$ is a knot with $\mathcal{A}_{t}(K)=\mathcal{A}_{t}\left(K^{\prime}\right)$ and $\kappa_{j}(K)=\kappa_{j}\left(K^{\prime}\right)$ for $j=1,2, \cdots, \nu(K)$, then $\kappa_{j}(K)=$ $\kappa_{j}\left(K^{\prime}\right)$ for all $j$. Moreover, $\nu(K)$ depends only on the noncommutative A-ideal of $K$.

Proof. In view of the previous theorem, the only thing we have to show is that the noncommutative ideal, or equivalently the peripheral ideal, is never zero. This is done as follows. Consider the map between skein modules $\phi: K_{t}\left(\mathbb{T}^{2} \times I\right) \rightarrow$ $K_{t}\left(S^{3} \backslash N(K)\right)$ induced by the inclusion of the cylinder into the knot complement (here $N(K)$ is a regular neighborhood of the knot). Then, as a submodule, $I_{t}(K)$ is the kernel of this map. If $I_{t}(K)=(0)$, then $\phi$ is an isomorphism onto the image.

On the other hand, if we add a 2-handle to the knot complement along the meridian of the knot, then the skein module of the knot complement, and hence the image of $\phi$, becomes the one dimensional free module (i.e. the structure ring $\left.\mathbb{C}\left[t, t^{-1}\right]\right)$. If we add a 2-handle to the cylinder over the torus along the meridian, the skein module becomes $\mathbb{C}\left[t, t^{-1}, \alpha\right]$, where $\alpha$ is the curve which is the image of the longitude, which is an infinite dimensional free module. But Hoste and Przytycki 7 have shown that adding a 2-handle induces a set of algebraic relations at the level of the skein module, in particular the same relations are introduced in $K_{t}\left(\mathbb{T}^{2} \times I\right)$ and in its image through $\phi$. Since adding these relations produces different modules, $\phi$ is not an isomorphism, so it has a kernel.

As the reader probably guessed, the working conjecture behind all this work is that the noncommutative A-ideal always determines the colored Kauffman brackets of the knot. The result below shows that this happens to be true in certain situations.

Theorem 2. Assume that $K$ is a knot with the property that $\mathcal{A}_{t}(K)$ contains a polynomial $\varphi=\sum_{p, q} \gamma_{p, q} l^{p} m^{q}$ of degree 2 in $l$ such that there exists no $n \geq 0$ for which the expression $\sum_{q}\left(\gamma_{2, q}+\gamma_{0, q}\right)(-1)^{q} t^{(2 n+2) q}$ is identically equal to zero. Then for any knot $K^{\prime}$ with the property that $\mathcal{A}_{t}(K)=\mathcal{A}_{t}\left(K^{\prime}\right)$, it follows that $\kappa_{n}(K)=\kappa_{n}\left(K^{\prime}\right)$ for all $n=1,2,3, \ldots$.

Proof. The polynomial $\varphi$ gives rise to an element $a=\sum_{i} c_{i}\left(1, q_{i}\right)_{T}+u$ in $I_{t}(K)$, with $c_{i}=t^{q_{i}}\left(\gamma_{2, q_{i}}+\gamma_{0, q_{i}}\right)$ and $u$ a polynomial in $(0,1)$. By Lemma $1, T_{n}(\alpha) \cdot u$ is of the form $\lambda S_{n}(\alpha)+\mu S_{n-2}(\alpha), \lambda, \mu \in \mathbb{C}\left[t, t^{-1}\right]$. On the other hand, the same lemma shows that

$$
\begin{aligned}
& T_{n}(\alpha) \cdot \sum_{i} c_{i}\left(1, q_{i}\right)_{T}=\sum_{i} c_{i}\left[(-t)^{(2 n+3) q_{i}} S_{n+1}(\alpha)\right. \\
& \left.-(-t)^{(2 n-1) q_{i}} S_{n-1}(\alpha)+(-t)^{(-2 n+3)} S_{1-n}(\alpha)-(-t)^{(-2 n-1) q_{i}} S_{-n-1}(\alpha)\right] .
\end{aligned}
$$

Since $S_{-k}=-S_{k-2}$ for all $k$, this is further equal to

$$
\begin{aligned}
\sum_{i} c_{i}\left[(-t)^{(2 n+3) q_{i}} S_{n+1}(\alpha)-\left[(-t)^{(2 n-1) q_{i}}\right.\right. & \left.+(-t)^{(-2 n-1) q_{i}}\right] S_{n-1}(\alpha) \\
& \left.+(-t)^{(-2 n+3) q_{i}} S_{n-3}(\alpha)\right] .
\end{aligned}
$$


Hence the orthogonality relation applied to $a$ yields a $\mathbb{C}\left[t, t^{-1}\right]$-linear equation in $\kappa_{n+1}(K), \kappa_{n}(K), \kappa_{n-1}(K), \kappa_{n-2}(K)$, and $\kappa_{n-3}(K)$. For $n \geq 1$, the coefficient of $\kappa_{n+1}(K)$ is $\sum_{i} c_{i}(-t)^{(2 n+3) q_{i}}$, and the condition from the statement translates to the fact that for no $n$ this is identically equal to zero.

On the other hand, for $n=0$, since $\kappa_{0}(K)=1, \kappa_{-1}(K)=0, \kappa_{-2}(K)=-1$, $\kappa_{-3}(K)=-\kappa_{1}(K)$, the orthogonality relation gives a linear equation in $\kappa_{1}(K)$. The coefficient of $\kappa_{1}(K)$ is $2 \sum_{i} c_{i}(-t)^{3 q_{i}}$. Again this is not equal to zero. So the equation can be solved uniquely for $\kappa_{1}(K)$.

Using $\kappa_{1}(K), \kappa_{0}(K)=1, \kappa_{-1}(K)=0$, and $\kappa_{-2}(K)=1$ as the initial condition, the orthogonality relation provides a recursion that uniquely determines $\kappa_{n}(K)$ from $\kappa_{1}(K)$. It follows that the A-ideal determines the colored Kauffman brackets of the knot, and we are done.

Observe that the degree in $l$ of any polynomial in $\mathcal{A}_{t}(K)$ is at least 2 .

\section{EXAMPLES}

The unknot. The A-ideal of the unknot is generated by $\left(l+t^{2}\right)\left(l+t^{-2}\right)$ and $l m^{2}\left(l+t^{2}\right)+t^{2}\left(l+t^{-2}\right) 5$; hence it satisfies the conditions in Theorem 2. The orthogonality relation for $\left(l+t^{2}\right)\left(l+t^{-2}\right)$, that is, for $(1,0)_{T}+t^{2}+t^{-2} \in I_{t}(K)$, gives

$$
\begin{gathered}
\kappa_{0}(K)=1, \quad \kappa_{1}(K)=-t^{2}-t^{-2}, \\
\kappa_{n+1}(K)=\left(-t^{2}-t^{-2}\right) \kappa_{n}(K)-\kappa_{n-1}(K), \quad n \geq 1 .
\end{gathered}
$$

Thus in this case the orthogonality relation is the recurrence for Chebyshev polynomials with variable replaced by $-t^{2}-t^{-2}$. We obtain

$$
\kappa_{n}(K)=(-1)^{n}[n+1]=(-1)^{n}\left(t^{2 n+2}-t^{-2 n-2}\right) /\left(t^{2}-t^{-2}\right) .
$$

The orthogonality relation for the other element leads to a different recursive relation with the same general term formula.

The trefoil. The A-ideal of the left-handed trefoil is generated by

$$
\begin{gathered}
{\left[m^{4}\left(l+t^{10}\right)-t^{-4}\left(l+t^{2}\right)\right]\left(l-t^{6} m^{6}\right),} \\
\left(l+t^{24}\right)\left(l+t^{10}\right)\left(l+t^{2}\right)\left(l-t^{6} m^{6}\right)
\end{gathered}
$$

and

$$
\left(m^{2}-t^{-22}\right)\left(l+t^{10}\right)\left(l+t^{2}\right)\left(l-t^{6} m^{6}\right)
$$

(see [6]). A quick look at the element $\left[m^{4}\left(l+t^{10}\right)-t^{-4}\left(l+t^{2}\right)\right]\left(l m^{6}-t^{6}\right)$ shows that the conditions in the statement of Theorem 2 are fulfilled. This element corresponds to

$$
(1,-5)_{T}-t^{-8}(1,-1)_{T}+t^{3}(0,5)_{T}-t(0,1)_{T}
$$

in the peripheral ideal. The orthogonality relation produces the following recursion

$$
\begin{gathered}
\left(-t^{-10 n-15}+t^{-2 n-11}\right) \kappa_{n+1}(K)+\left(-t^{10 n+7}-t^{-10 n-13}+t^{2 n+3}\right. \\
\left.+t^{-2 n-1}\right) \kappa_{n}(K)+\left(t^{-10 n+5}-t^{10 n+5}-t^{-2 n-7}+t^{2 n-7}\right) \kappa_{n-1}(K)+\left(t^{10 n-13}\right. \\
\left.+t^{-10 n+7}-t^{2 n-1}-t^{-2 n+3}\right) \kappa_{n-2}(K)+\left(t^{10 n-15}-t^{2 n-11}\right) \kappa_{n-3}(K)=0 .
\end{gathered}
$$


In particular, for $n=0$,

$$
\left(t^{-11}-t^{-15}\right) \kappa_{1}(K)-t^{7}-t^{-13}+t^{3}+t^{-1}=0
$$

and hence $\kappa_{1}(K)=t^{18}-t^{10}-t^{6}-t^{2}$, the well known formula for the Kauffman bracket of the trefoil knot with framing zero.

\section{REFERENCES}

1. C. Blanchet, N. Habegger, G. Masbaum, P. Vogel, Topological quantum field theories derived from the Kauffman bracket, Topology 31(1992), 685-699. MR 96i:57015

2. D. Bullock, Rings of $\mathrm{SL}_{2} \mathbb{C}$-characters and the Kauffman bracket skein module, Comment. Math. Helv. 72(1997), no. 4, 521-542. MR 98k:57008

3. D. Cooper, M. Culler, H. Gillett, D.D. Long, P.B. Shalen, Plane Curves associated to character varieties of 3-manifolds, Inventiones Math. 118(1994), pp. 47-84. MR 95g:57029

4. C. Frohman, R. Gelca, Skein Modules and the Noncommutative Torus, Trans. Amer. Math. Soc. 352(2000), 4877-4888. MR 2001b:57014

5. C. Frohman, R. Gelca, W. Lofaro, The A-polynomial from the noncommutative viewpoint, preprint.

6. R. Gelca, Noncommutative trigonometry and the A-polynomial of the trefoil knot, preprint.

7. J. Hoste, J.H. Przytycki, The $(2, \infty)$-skein module of lens spaces; a generalization of the Jones polynomial, J. Knot Theor. Ramif., 2(1993), no. 3. 321-333. MR 95b:57010

8. V.F.R. Jones, Polynomial invariants of knots via von Neumann algebras, Bull. Amer. Math. Soc., 12(1985), 103-111. MR 86e:57006

9. L. Kauffman, States models and the Jones polynomial, Topology, 26(1987), 395-407. MR 88f:57006

10. W.B.R. Lickorish, The skein method for three-manifold invariants, J. Knot Theor. Ramif., 2(1993) no. 2, 171-194. MR 94g:57006

11. J. H. Przytycki and A. Sikora, Skein algebra of a group, Banach Center Publ. 42. MR 99e:57019

12. N.Yu. Reshetikhin, V.G. Turaev, Invariants of 3-manifolds via link polynomials and quantum groups, Inventiones Math., 103(1991), 547-597. MR 92b:57024

13. V.G. Turaev, Quantum invariants of Knots and 3-manifolds, de Gruyter Studies in Mathematics, de Gruyter, Berlin-New York, 1994. MR 95k:57014

Department of Mathematics and Statistics, Texas Tech University, Lubbock, Texas 79409 - and - Institute of Mathematics of the Romanian Academy, Bucharest, Romania

E-mail address: rgelca@math.ttu.edu 